\title{
Delivering Group-Based Services to the Home via the Internet: Maximising Clinical and Social Benefits
}

\author{
Andrea Taylor, Richard Wilson \\ Centre for Design Innovation \\ Glasgow School of Art \\ Forres, Scotland
}

\author{
Angus Aitken \\ New Products and Platforms \\ LifeScan \\ Inverness, Scotland
}

\author{
David Godden \\ Centre for Rural Health \\ University of Aberdeen \\ Inverness, Scotland \\ Judith Colligan \\ Physiotherapy Department \\ NHS Highland \\ Inverness, Scotland
}

\begin{abstract}
Delivering clinical services to people in their homes by means of technology may improve access for patients, but may compromise personal and social interactions with health professionals. Rehabilitation programs improve wellbeing and quality of life in a number of conditions, such as Chronic Obstructive Pulmonary Disease (COPD), Ischemic Heart Disease and Stroke. Programs are conventionally delivered to groups, who undertake shared physical exercise, education and socialization. However, travel demands or poor health mean that many patients cannot participate. In a previous paper, we reported a new design for a group pulmonary rehabilitation program delivered to the home via the Internet and videoconferencing, and reported findings from the first feasibility trial performed with four patients. A second feasibility trial has been performed with three patients. Across both trials, the technology worked well, patients found the system easy to use, felt part of a group and felt safe. Satisfaction was high and clinical outcomes were similar to those in a conventional clinicbased program. Limited social interaction between participants occurred. In future, this could be enhanced by modifying the program schedule to dedicate time for socialization, and by allowing use of the system for interaction outwith the scheduled sessions. The technology could potentially be applied to other forms of rehabilitation such as Ischemic Heart Disease and Stroke.
\end{abstract}

Keywords-Chronic Obstructive Pulmonary Disease (COPD); health care; Internet; physical exercise; pulmonary rehabilitation; rehabilitation; socialization; videoconferencing.

\section{INTRODUCTION}

Determinants of physical and mental health are multifactorial, influenced not only by genetic and environmental factors, but also by personal and social contact, which has positive impacts on older peoples' feelings of wellbeing [1]. However, social networks diminish with age and older people can experience loneliness and depression as friends and families may live a considerable distance away [2, 3]. Rural older people may be further isolated due to migration patterns or existing co-morbidities such as chronic pain $[4,5]$.
Delivering care and rehabilitation services to rural older people is challenged by geography, and policymakers see technology as a potential solution. However, older adults have indicated that their greatest fear with the growing use of technologies in their care is losing personal and social interaction with nurses and other community-based service providers - this interaction is central to their healthcare experience [6,7]. Willingness to accept technology may be influenced by health status. A large scale US survey demonstrated that as people become ill, they become more willing to accept monitoring, but only if it does not reduce social contact [8].

Rehabilitation has become increasingly important as a strategy to improve wellbeing and quality of life in a number of conditions, such as Chronic Obstructive Pulmonary Disease (COPD), Ischemic Heart Disease, Stroke, and following trauma. In some cases, rehabilitation will be specifically tailored for an individual patient and will be delivered on a one to one basis. However, for other conditions, such as COPD and Ischemic Heart Disease, rehabilitation programs are delivered in a group setting and there is evidence that participation in the group is an important determinant of the outcome of the program [9]. Conventionally, COPD rehabilitation groups will meet on a regular basis at a defined location, usually a hospital or other clinical facility, and will be directly supervised by a physiotherapist. At each class, in addition to a supervised exercise program, the patients will be offered a series of educational talks about issues such as smoking cessation or inhaler technique [10], and in many cases there will also be a social element to the class, sharing refreshments and informal chat at the end of the session. The effectiveness of conventional pulmonary rehabilitation is established [11]. However, patients who are unable to attend a central hospital or clinic facility by virtue of geography, lack of transport or poor health are denied both the clinical and social benefits of participation.

In a previous paper, we described our prototype design for a group pulmonary rehabilitation program delivered to the home via the Internet and videoconferencing [12]. We reported

The study was funded by Chest, Heart and Stroke Scotland. 
findings from the first trial demonstrating the feasibility of delivering such a program, with clinical outcomes comparable to a conventional program. This paper contributes findings from the second trial with a discussion of the main challenges encountered, and opportunities for future development of such systems. The paper is organized as follows. We first review related work on delivering pulmonary rehabilitation at a distance. For context, we briefly summarize the research methods. Thereafter, we report the combined findings of the two trials: feasibility, acceptability, clinical effectiveness, and challenges of delivering pulmonary rehabilitation to the home via the Internet and videoconferencing. We conclude with a discussion of the wider potential of such systems.

\section{RELATED WORK}

This section provides examples of three approaches to the remote delivery of pulmonary rehabilitation.

\section{A. Centre-Based Pulmonary Rehabilitation}

Two programs, which use satellite centres closer to patients' homes than the main hospital, have reported their findings. Tayside National Health Service (NHS) Board in Central Scotland delivered a program to three patients at a remote community hospital, using high-end videoconferencing linked to a conventional class at the main hospital centre [13]. Patient satisfaction was high. A comparable program is run as part of routine clinical care at the Centre for Lung Health in Western Canada [14]. High-end videoconferencing equipment is used to link 8-12 patients at the Centre with 2-6 patients in 10 satellite groups at remote hospitals or health centers. In both programs clinical outcomes were reported as comparable to those from the standard pulmonary rehabilitation program at the main Centre. However, both programs still require patients to travel to a clinic location to participate, and involve a clinician at the remote site to supervise and operate the equipment. Also, videoconferencing is used for the education component of the programs only, not for supervision of exercises.

\section{B. Self-Managed Pulmonary Rehabilitation}

In China, a mobile phone application was developed that delivered music at a variable tempo as a background to a tailored walking program over one year [15]. The results indicated good compliance and clinical outcomes, but there was no group or social element to the program. Reference [16] also uses a mobile phone application, combined with a pulse oximeter (a device that measures heart rate and blood oxygen saturation), for self-management of pulmonary rehabilitation. Heart rate and time remaining are displayed on the screen during exercise; color-coding and a siren-like sound alert the patient if their heart rate exceeds safe limits. The data, including blood oxygen saturation, are saved to the phone memory card for upload to a PC for a clinician to examine. However, this approach also lacks the group setting and peer support of conventional programs.

\section{Home-Based Pulmonary Rehabilitation}

The design and implementation of a Norwegian pulmonary rehabilitation system, involving a dedicated PC running open

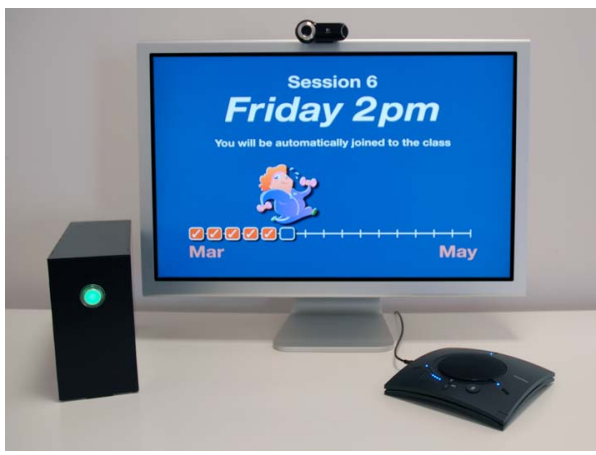

Figure 1. A custom designed PC was connected to each patient's home TV and wired to the Internet, together with a webcam, speakerphone, and where appropriate, a wireless pulse oximeter.

source videoconferencing software connected to the patient's home TV and the Internet, has been published [17], but there have to date been no reports of clinical outcomes or discussion of any social or other benefits from this system.

\section{METHODS OVERVIEW}

Our methods and prototype design for a group pulmonary rehabilitation program delivered to the home via the Internet and videoconferencing, is described in [12]. A brief summary for context follows.

\section{A. Design and Technology}

The design was informed by initial discussions with a patient group, described in [12]. A mini PC was re-boxed in a simple case with a single large on/off button, to create an easy interaction (Fig. 1). The PC was connected to the patient's home TV and wired to the Internet, together with a webcam (Logitech QuickCam Pro 9000), speakerphone (ClearOne Chat 150 USB), and, where appropriate, a wireless pulse oximeter (Nonin Onyx II, 9560). The physiotherapist was equipped with a desktop PC wired to the Internet and connected to two video screens, the same webcam and speakerphone, and a standard CD player. A 40-inch screen displayed the video. Positioned beneath, a 20-inch screen displayed patient information including pulse oximeter readings and an exercise timer. To create a group spirit, video screen layouts were selected that displayed all the participants simultaneously (Fig. 2).

A videoconferencing service (JVCS Desktop) available to the JANET community was employed, which enables 12 users

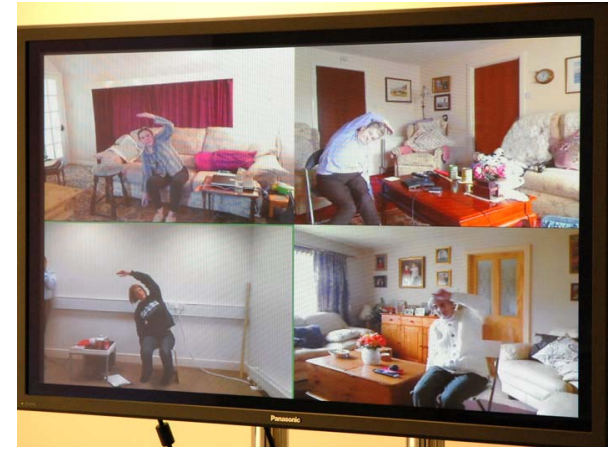

Figure 2. The optimum video screen layout was selected that displayed all the participants - in this instance, the physiotherapist appeared bottom left. 


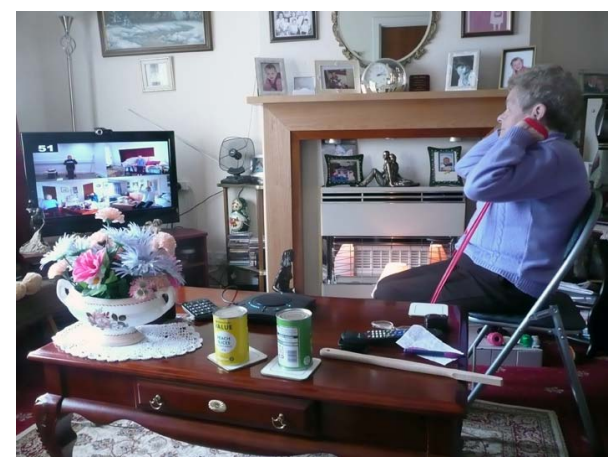

Figure 3. The exercises used resistive elastic bands and everyday household objects in place of specialized gym equipment e.g. tinned fruit as arm weights

to dial in to a videoconference from a Windows-enabled PC running free software by Tandberg called ConferenceMe [18]. In addition, custom software was developed to: make it very easy for patients to join a conference, time the exercises, and communicate pulse oximeter data to the physiotherapist in realtime. The software was developed using Microsoft's .NET framework and written in $\mathrm{CH}$. It used a client-server model where the physiotherapist's PC was the server and the patients' PCs the clients. The server sent commands to the clients using Internet Protocol Technology (TCP/IP) on a static IP address and read data back over the Internet; the clients continuously listened on an IP port for incoming commands. ConferenceMe and the custom software ran independently.

\section{B. $\quad$ Procedure and Outcome Measures}

Two feasibility trials, approved by The North of Scotland Research Ethics Service, were performed in our NHS Board area of Highland in 2010, with four and three patients respectively: three males and four females, aged 58-79, with moderate to severe COPD. A physiotherapist supervised exercises and advice sessions from the research lab; similar to those delivered at a conventional clinic-based program (Fig. 3). Patients joined the class by switching their TV to the video channel. During classes, all participants could see and hear each other. The physiotherapist could also monitor individual patients' blood oxygen saturation. Classes took place twice weekly for eight weeks.

Pre- and post- program clinical assessment included: Chronic Respiratory Questionnaire (CRQ) [19] and Hospital Anxiety and Depression Scale (HADS) [20] to assess breathing difficulties and general wellbeing; incremental shuttle-walking test to assess exercise capacity [21]; and blood oxygen saturation measurements. Patients were asked to complete an anonymous satisfaction questionnaire developed by the project team, together with the more generic Client Satisfaction Questionnaire (CSQ) [22]. The physiotherapist also completed a brief satisfaction questionnaire.

\section{RESULtS}

\section{A. Program Compliance}

All patients completed the program. The average attendance rate was $78 \%$ (range $63 \%-100 \%$ ), which is comparable to conventional programs [e.g. 23]. Non-attendance was mostly due to medical reasons e.g. exacerbations (worsening of COPD symptoms), hospitalizations, and medical appointments. Other reasons included no electricity at home due to local authority works and TV repair. One patient missed a class due to a temporary problem connecting to the Internet.

\section{B. Reliability of the Technology}

Patients encountered few problems operating the system; in trial two, one patient needed instruction by telephone to switch to the video channel for the first two sessions. Overall, the audio and video quality was acceptable. Some audio problems were experienced in one-third of the sessions during trial one Tandberg later revised ConferenceMe and the situation did not recur in trial two - and a slight audio delay made engaging in conversation more challenging. One patient occasionally experienced low-quality video because of slow broadband speeds. As speeds improve, this should be overcome. Another experienced intermittent Internet loss, possibly because of electrical interference or very poor quality in-home wiring. Five patients were issued with a pulse oximeter. The single usability factor was curling the finger to read the values; one patient initially read the digits upside down (e.g. ' 3 ' as 'E'). In trial one, the occasional reading was not displayed on the physiotherapist's screen, in which case patients reported readings verbally. In trial two, technical problems rendered automatic transmission of oxygen saturation and pulse rate data to the physiotherapist less reliable and patients were often required to report readings verbally.

\section{Level of Satisfaction}

Patients reported that they had felt comfortable (4) or very comfortable (3) with taking part by videolink before the program and nearly all (6) were very comfortable after the program. Patients reported watching the physiotherapist during the exercises some of the time (1), most of the time (4) or all of the time (2). While most reported watching their class members some of the time (3), most of the time (1) or all of the time (1), two reported watching the physiotherapist only. However, all reported that they felt part of a group and felt safe. All found the program beneficial, and nearly all (6) were able to control their breathing more easily after the program. While one reported their way of life improved considerably, others reported a little improvement (4) or no change (2). However most (5) reported that they went out more often for social purposes after the program. Generic satisfaction scores on value of service received were high: CSQ values 29-32. ( $32=$ maximum possible score). All reported that they would definitely come back to the program if they were to seek help again, and that they would recommend the program to a friend in need of similar help.

The physiotherapist felt comfortable using the system. However, one weakness of the system was the inability to have private one-to-one conversations with individual participants during the sessions. Issues requiring this form of contact involved a separate telephone contact.

\section{Clinical Effectiveness}

Incremental shuttle-walking distances improved in four patients by at least 50 meters; the minimal clinically important 
difference (MCID) is 48 meters. One patient did not attend for shuttle-walking tests due to distance of travel. Of the remaining two: distance increased by just 30 meters in one patient; and fell by 80 meters in the other, likely because of a chest infection at the time of the follow-up test. Overall, the mean difference in incremental shuttle-walking distances was 50 meters, which is comparable to 42 meters among patients $(n=26)$ undergoing the program at the main hospital serving the region, Raigmore, in 2010.

All patients' CRQ scores improved in at least one domain: dyspnoea (5), emotion (6), fatigue (6) and mastery (5). The mean change was: dyspnoea 4.3, emotion 5.3, fatigue 2.6, mastery 2.0. HADS scores improved in six patients and worsened in one patient. The mean change was: anxiety -2.1 , depression -2.1 (in the case of HADS, negative scores represent improvement). Again, the results are comparable to the mean change in patients $(\mathrm{n}=26)$ undergoing the hospital-based program in 2010: CRQ - dyspnoea 4.3, emotion 1.6, fatigue 2.3, mastery 1.7; HADS - anxiety +1.1 , depression -0.1 .

\section{E. Social Interaction}

Patients reported that they did feel part of the group, and on occasions when people were on-line but the class had not formally commenced, we did observe patients interacting with each other. There were two issues associated with all patients being on video and audio simultaneously. First, there is a need to adopt etiquette for conversations to avoid overlapping due to the effects of latency in the connections. Second, as observed by the physiotherapist, it is impossible to conduct a one-to-one confidential discussion with the present configuration. We are unaware if the patients in the group established social relationships outwith the class, as does occur with conventional programs. It may be appropriate in future studies to create systems to facilitate such interaction, as discussed later.

\section{Challenges}

\section{A. Patient Recruitment}

The aim was to recruit up to eight patients for each trial, based on a staff/patient ratio guideline in the UK of 1:8 for exercise classes [10]. However, recruitment of patients was very challenging: only one in three of those approached agreed to participate. In trial one, 12 patients referred for pulmonary rehabilitation by their hospital consultant were contacted, of whom eight declined to participate. In trial two, we contacted around 10 patients referred by their hospital consultant or general practitioner. Recruitment was even more challenging and due to time constraints, we opted to proceed with the three patients who had agreed to participate. Nearly all of those who declined to participate in the home-based program also declined conventional pulmonary rehabilitation, despite having previously accepted referral. Some gave specific reasons e.g. current admission to hospital and moving away from the area. On five occasions, the session took place with only one patient.

The recruitment difficulties were unexpected and not wholly explained, although they may have been partly related to the large volume of paperwork that patients had to read through (patient information, consent forms etc.) as required for Ethical Approval as this was a clinical trial. We speculate that if this mode of delivery were simply offered as a clinical service, uptake might be higher. Also, most conventional pulmonary rehabilitation programs operate on a rolling basis i.e. patients are entering and leaving at different times, as opposed to our program, where they all started at once and all finished at once for logistical reasons. Recruitment might be improved with a rolling program with flexible start dates, although the group dynamics would be changed.

\section{B. Broadband Ownership}

A significant challenge to NHS Boards, including Highland, in adopting a home-based rehabilitation model is the temporary provision of broadband to homes that do not already have it and the actual speeds available. A recent survey by Ofcom, the UK's communications regulator, put Scotland as the least connected nation in the UK with just $61 \%$ of the population owning broadband, with the highest level of nonadoption for involuntary reasons - lack of availability and cost [24]. While most homes in Scotland do have access to a fixedbroadband service, long line lengths and/or lines of insufficient quality between homes and the local telephone exchange can compromise speeds and inhibit Internet use. In addition, [24] found that speeds typically decreased with distance from the exchange, a problem most likely to arise in rural areas, and that the proportion of lines incapable of delivering download speeds of $2 \mathrm{Mbit} / \mathrm{s}$ was higher in the Highlands and Islands - upload speeds are usually a lot slower than the advertised download speed. However, funding has just been allocated by the UK Government to provide superfast broadband across the Scottish Highlands and Islands that will expedite and boost the use of new technology in rural healthcare delivery.

Four patients required installation of a broadband Internet connection. Consistent with [24], which found Internet take-up higher among younger people than older people, the youngest two patients had Internet access at home. The cost of taking out 12-month broadband contracts (BT Total Broadband Option 1) was met by the project budget. When the program ended, the broadband accounts were closed incurring an anticipated charge for ending the service early. However, while broadband Internet service providers have traditionally required a minimum 12-month contract term, much longer than the eightweek program, short-term contracts are starting to emerge. In addition, while use of $3 \mathrm{G}$ technologies was not considered due to low coverage in the Highlands and Islands, this method would avoid the issues of administering individual broadband accounts and fixed-term contracts.

\section{CONCLUSION AND FUTURE WORK}

This is the first study in the UK to deliver group-based pulmonary rehabilitation to patients in their homes. In general, the technology worked well despite relatively slow broadband speeds and patients found the system easy to use. The sample size is small; nonetheless all patients completed the program, patient satisfaction scores were high, and attendance rates and clinical outcomes were similar to those of patients attending a conventional centre-based program. The preliminary results indicate that this method of delivery offers potential to deliver group-based rehabilitation to patients who are unable to attend 
conventional classes. Having demonstrated feasibility, testing the intervention on a larger scale would now be appropriate. The technology could potentially be adapted for other clinical scenarios e.g. Ischemic Heart Disease or Stroke.

Some examples of potential future work that could be applied to other rehabilitation programs are:

- Measuring Performance. In a conventional pulmonary rehabilitation program, patients count the number of repetitions they perform per exercise, which staff manually record and use as the basis for gradually increasing the training intensity. Developing a system of automatically logging repetitions by individual patients, by means of an ankle and wrist cuff with motion sensing capability, could improve accuracy and simplify the process for the patient.

- Affecting Performance. People with COPD often stop exercising due to anxiety over becoming more breathless. A modified Borg Scale (0-10) allows patients to grade the intensity of their breathlessness during exercise and can motivate effort [10]. In a conventional class, patients call out their Borg scores. A numeric keypad connected to the patient's PC for sending scores to the physiotherapist, would give privacy and could remove the potential for other group members to influence individual patients' scores.

- Exercise Summary. A sense of achievement or progress can be motivational. In a conventional pulmonary rehabilitation program, patients need to request information on performance from staff. Developing an exercise summary page customized to each individual that can be viewed at leisure on the home TV, could encourage interest and attendance and improve attitudes towards exercise.

- Enhancing Personal and Social Interaction. A number of opportunities arise to enhance personal and social interaction. First, the duration of sessions could be increased to specifically incorporate a social period. Adaptation of the technology to allow patients to videoconference with each other for social purposes on a one-to-one basis or as a group outwith the rehabilitation sessions could be explored. Finally, opportunities for patients to link with each other or with their health professional outside of the sessions using other media could be explored.

\section{ACKNOWLEDGMENT}

We thank Alan Sloan at JANET Videoconferencing Service (JVCS) and David Powell for technical assistance. We also thank NHS Highland patients who volunteered to take part in the study and Corinne Clark for delivering education talks.

\section{REFERENCES}

[1] G. C. Wenger, "Social networks and the prediction of elderly people at risk," Aging \& Mental Health, 1997, vol. 1, pp. 311-320.

[2] J. J. Gierveld, "A review of loneliness: concept and definitions, determinants and consequences," Reviews in Clinical Gerontology, 1998, vol. 8, pp. 73-80.
[3] J. E. Lansford, A. M. Sherman, and T. Antonucci, "Satisfaction with social networks: an examination of socio-emotional selectivity theory across cohorts," Psychology \& Aging, 1998, vol. 13, pp. 544-552.

[4] A. Kumar and N. Allcock, "Pain in older people: reflections and experiences from an older person's perspective," 2008, http://www.britishpainsociety.org/book_pain_in_older_age_ID7826.pdf.

[5] M. Shucksmith and L. Philip, "Social exclusion in rural areas: a literature review and conceptual framework," 2000, http://www.scotland.gov.uk/Publications/2000/08/9588/File-1.

[6] J. Farmer et al., "Territorial tensions: stakeholder perspectives on health services for rural older people," Health and Place, 2010, vol. 16, pp. 275-283.

[7] C. Potter, "What quality healthcare means to older people: exploring and meeting their needs," Nursing Times, 2009, vol. 105, pp. 14-18.

[8] S. Beach et al., "Disability, age, and informational privacy attitudes in quality of life technology applications: results from a national web survey," ACM Transactions on Accessible Computing, 2009, vol. 2:5, pp. $1-21$.

[9] S. D. O’Shea, N. F. Taylor, and J. D. Paratz, “...But watch out for the weather: factors affecting adherence to progressive resistance exercise for persons with COPD," Journal of Cardiopulmonary Rehabilitation and Prevention, 2007, vol. 27, pp. 166-74.

[10] M. D. L. Morgan, "Pulmonary rehabilitation. British Thoracic Society standards of care subcommittee on pulmonary rehabilitation," Thorax, 2001, vol. 56, pp. 827-834.

[11] Y. Lacasse, R. Goldstein, T. J. Lasserson, and S. Martin, "Pulmonary rehabilitation for chronic obstructive pulmonary disease," Cochrane Database of Systematic Reviews, 2006, issue 4, Art no:CD003793, DOI 10,1002/14651858.CD003793.pub2.

[12] A. Taylor, A. Aitken, D. Godden, and J. Colligan, "Group pulmonary rehabilitation delivered to the home via the Internet: feasibility and patient perception," In Proc. CHI 2011, in press.

[13] Scottish Centre for Telehealth, "Tele-pulmonary rehabilitation," 2009, http://www.sct.scot.nhs.uk/pulmonaryrehab.html.

[14] A. Holland, "The Jack Brockhoff Foundation Churchill Fellowship to discover new ways to deliver pulmonary rehabilitation for Australians with chronic lung disease," 2009, http://www.churchilltrust.com.au/site_media/fellows/2009_Holland_An ne.pdf.

[15] W. T. Liu et al., "Efficacy of a cell phone-based exercise programme for COPD," European Respiratory Journal, 2008, vol. 32, pp. 651-659.

[16] A. Marshall, O. Medvedev, and A. Antonov, "Use of a smartphone for improved self-management of pulmonary rehabilitation," International Journal of Telemedicine and Applications, vol. 2008, 2008, pp. 1-5.

[17] T. M. Burkow et al., "An easy to use and affordable home-based personal eHealth system for chronic disease management based on free open source software," Studies in Health Technology and Informatics, 2008, vol. 136, pp. 83-88.

[18] JANET, "Desktop videoconferencing", http://www.ja.net/services/video/jvcs/desktop/.

[19] J. E. Williams, S. J. Singh, L. Sewell, G. H. Guyatt, and M. D. Morgan, "Development of a self-reported Chronic Respiratory Questionnaire," Thorax, 2001, vol. 56, pp. 954-959.

[20] R. P. Snaith, "The Hospital Anxiety and Depression Scale," Health and Quality of Life Outcomes, 2003, vol. 1, p. 29.

[21] S. J. Singh, M. D. Morgan, S. Scott, and D. Walters, A. E. Hardman, "Development of a shuttle walking test of disability in patients with chronic airways obstruction," Thorax, 1992, vol. 47, pp. 1019-1024.

[22] D. L. Larsen, C. C. Attkisson, W. A. Hargreaves, and T. D. Nguyen, "Assessment of client/patient satisfaction: development of a general scale," Evaluation and Program Planning, 1979, vol. 2, pp. 197-207.

[23] R. Garrod, J. Marshall, E. Barley, and P. W. Jones, "Predictors of success and failure in pulmonary rehabilitation," European Respiratory Journal, 2006, vol. 27, pp. 788-94.

[24] Ofcom, "The Communications Market 2010: Scotland," 2010, http://stakeholders.ofcom.org.uk/market-data-research/marketdata/communications-market-reports/cmr10/scotland/. 